\title{
Data Mining by Using Rule of Space Syntax Theory
}

\author{
Shahla H.A. Kharofa \\ Department of Dental Basic Sciences, College Of Dentistry, Mosul University \\ Shahlakharofa@uomosul.edu.iq \\ DOI: $10.33899 /$ edusj.1970.163334
}

Received

18/ 02 / 2018

\author{
Accepted \\ $06 / 05 / 2018$
}

\begin{abstract}
Data mining is the technique, methods and means of extracting information from data and exploiting it to solve problems and make appropriate decisions using computer applications, which is a smart modern technology.

In this research, data mining and spaces syntax theory were used, a program was carried out to determine the structural importance of the spaces to determine the degree of their objective importance and comprehensiveness at the level of the system as a whole, school of two floors was selected and used for analyzing. Matlab language was used to perform the analysis.

Through applying the program, integration kernel showed the most integrated spaces locations which have the lowest degrees Real Relative Asymmetry while the isolation kernel has showed the lowest integrated spaces which have the highest degrees Real Relative Asymmetry. The results were compatible with the logical locations which hypothesizes the space system integration geometrically.
\end{abstract}

Keywords: Data mining, Spaces syntax theory, Sequential analysis. 


\title{
التنقيب عن البيانات باستخلام نظرية قواعد تركيب الفضاءات
}

\author{
شهلة حازم احمد خروفة

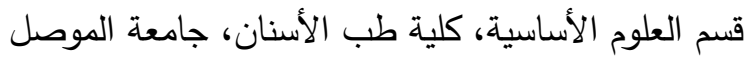

Shahlakharofa@uomosul.edu.iq

DOI: $10.33899 /$ edusj.1970.163334

(القبول

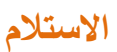

$2018 / 05$ / 06

$2018 / 02 / 18$

\section{الخلاصة}

التتقيب عن البيانات هي تقنيات وطرائق ووسائل لاستخلاص المعلومات من البيانات واستغلالها في

حل المشاكل واتخاذ القرارات المناسبة باستعمال تطبيقات الحاسبات والتي تعد تكنولوجيا حديثة ذكية.

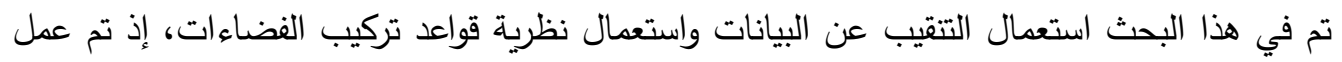

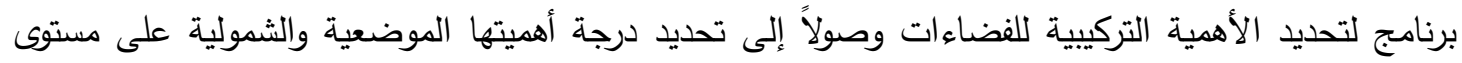

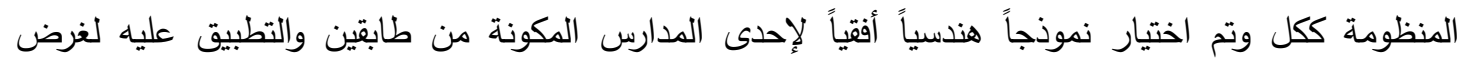
التحليل، وتم استعمال لغة ماثلاب لغرض إجراء عملية التحليل.

ومن خلال تطبيق البرنامج أظهرت نواة التكامل مواقع الفضاءات الأكثر تكاملاً والتي تحمل أوطأ

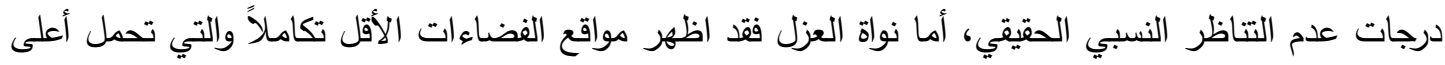

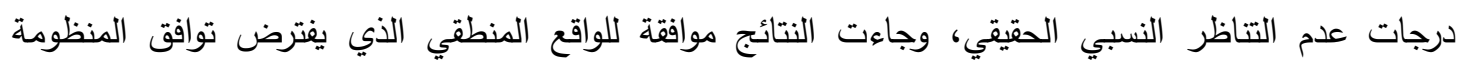
الفضائية على المستوى الهندسي. وتم عمل برنامج بلغة ماثلاب لتتفيذ عملية التحليل. الكلمات المفتاحية: التتقيب عن البيانات، نظرية تركيب الفضاءات، التحليل التسلسلي.

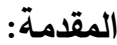

إن التطور في العلم والاقتصاد وتكنولوجيا المعلومات والاتصالات أدى إلى زيادة كمية البيانات الرقيـة

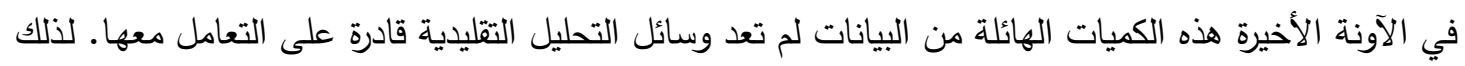

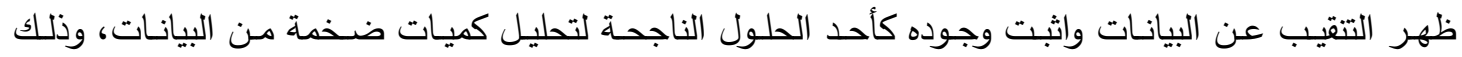
بتحويلها من مجرد معلومات متراكمة وغير مفهومة (بيانات) إلى معلومات قيمة يمكن استغلالها و الاستفادة منها بعد ذلك. [1] وقد ظهرت الحاجة إلى تجميع البيانات للاستفادة منها في أواخر الخمسينيات، وتبعاً لذلك ظهرت قواعد البيانات في الستينيات كقوالب لتجميع البيانات ثم بعد ذلك ظهور أنظمة لتلك القواعد، وفي السبعينيات ظهرت نظم إدارة قواعد البيانات والتي كانت عبارة عن أنظمة التواصل بين قواعد البيانات المختلفة والمتباعدة وربطها معاً في شبكة واحدة لتسهل إدارتها، وظهر مصطلح التتقيب في البيانات في نهاية الثمانينات، وهو يجمع ما بين

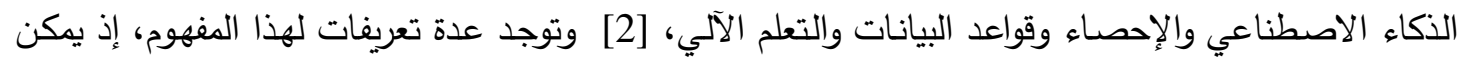

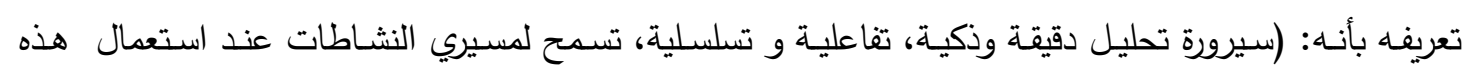




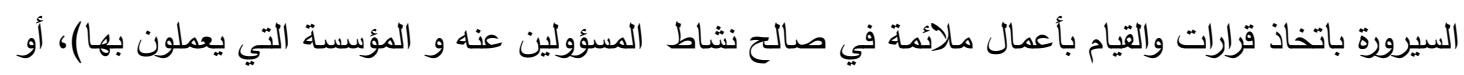
انه: (الاستكثاف الآلي لأنماط شائقة و غير جلية مخفية في قاعدة بيانات معينة) أو أنه: (عملية تحليل كمية فئة بيانات عادة مـا تكون كميـة كبيرة لإيجاد علاقة منطقيـة تلخص البيانات بطريقة جديدة تكون مفهومـة ومفيدة لصاحب البيانات). يطلق اسم نماذج (models) على البيانات والعلاقات الملخصة التي يتم الحصول عليها من التتقيب في البيانات. [3]

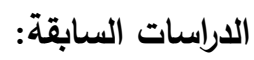

هناك المئات من الدراسـات حول التتقيب عن البيانات، لكنها تركز في الغالب على الأعمال التجاريـة

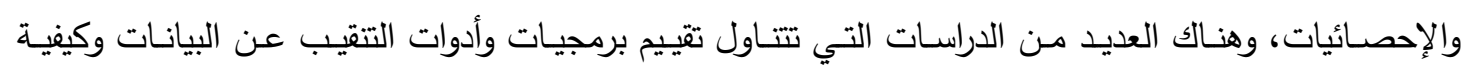
اختيارها. سيتم ذكر بعض الدراسات التي تتناول تقييم برمجيات وأدوات التتقيب عن البيانات: هنة 1999 قدم (Collier, K. ) كيفية استخدام التتقيب عن البيانات في السيطرة على اتجاه ومعلومات صنع القرار حول الممارسة المهنية و ممكن أن تستخدم كطريقة ومرجع لاختيار أفضل أداة

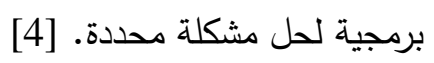
هنة 2004 قدمَ (Qiu, M. Davis) تقييم أدوات التتقيب عن البيانات التجارية لبيئة محددة من

$$
\text { [5] المستفيدين. }
$$

هنة 2010 قدم ( Androni, M.) دراسة بعض من أدوات التتقيب عن البيانات التجارية المتاحة مع بعض الاعتبارات التي تتعلق بتقييم أدوات التتقيب بواسطة الثركات التي ترغب في الحصول عن أتلى مثل

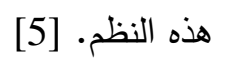
سنة 2014 قدم ( Lyras, D. ) استخدام وسائل وتقنيات التتقيب عن البيانات التعليمية في الدراسة

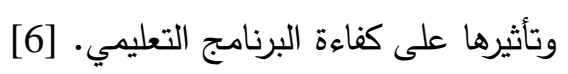
وعملية التتقيب عن البيانات تسمى أحياناً اكتثاف المعرفة وهي عملية تحليل البيانات من منظورات الئات مختلفة واستخلاص علاقات بينها وتلخيصها إلى معلومات مفيدة تقنياً.

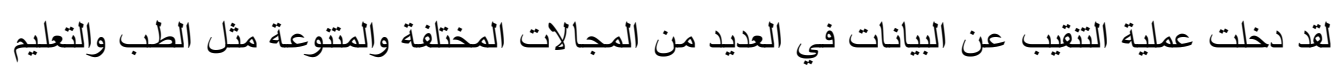

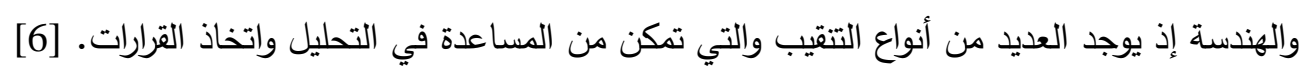
النقاط التي تعتمد عليها عملية التنقيب عن البيانات: ومن خلال ما سبق يمكن القول أن عملية التتقيب عن البيانات تعتمد على ما يلي: [9][8][7] 1. البيانات Data: هي عبارة عن الحقائق والأرقام والنصوص التي يمكن أن تعالج من قبل الحاسب.

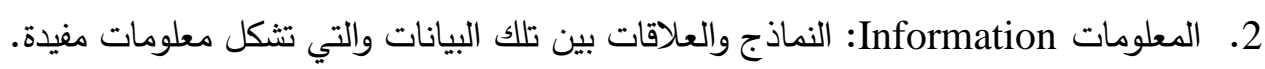
3. المعرفة Knowledge: المعلومات السابقة يمكن أن تحول إلى معرفة حول الأنماط المستعملة، فيساعدنا ذلك في معرفة أي من المواد سوف يتم احتياجها أكثر من غيرها. 4. مستودعات البيانات Data warehouse :المستعملة في التحليلات الزمنية واكتشاف المعرفة واتخاذ فيرهاذ

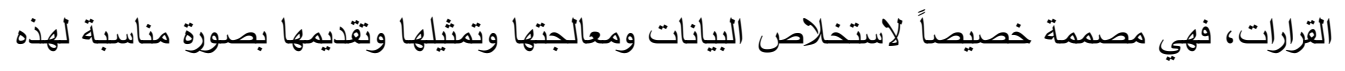
الأغراض، وتخزن كمية ضخمة من البيانات قد تكون من مصادر مختلفة. 
أنواع التنقيب:

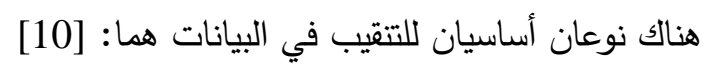

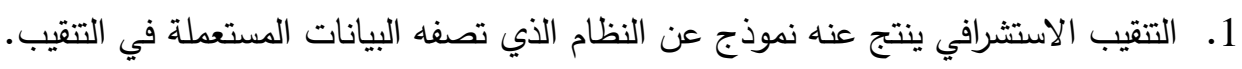
2. التتقيب الوصفي فينتج عنه معلومات جديدة بناء على المعلومات الموجودة داخل البيانات المستعملة في عملية التنقيب.

$$
\text { ميزات التنقيب عن البيانات: [12][11] }
$$

التقيب عن البيانات هو عمليـة تحليل البيانات(عادة مـا تكون كميـة كبيرة) من منظورات مختلفة

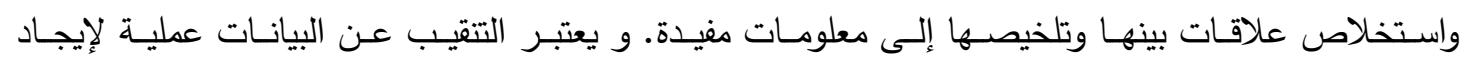

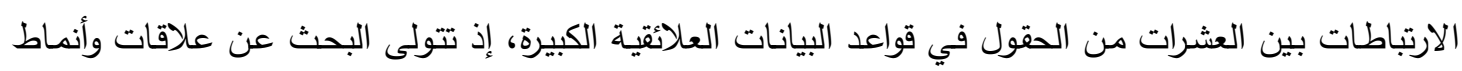

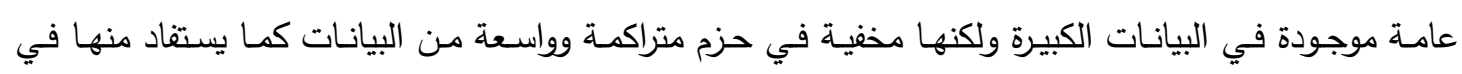

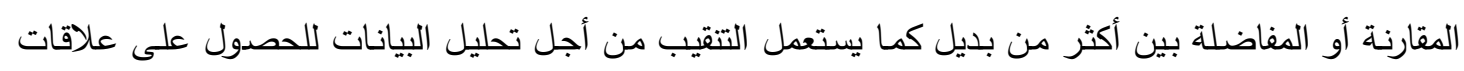

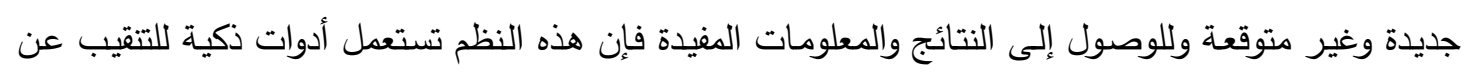

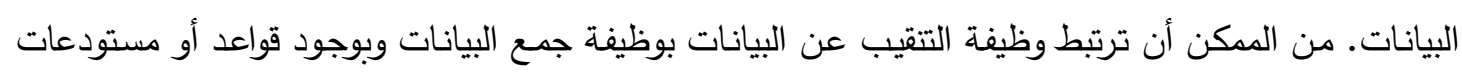

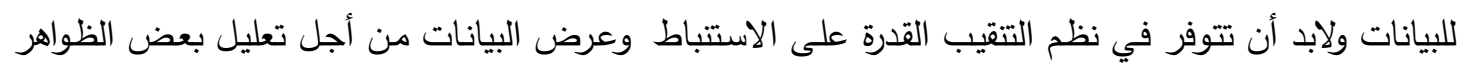

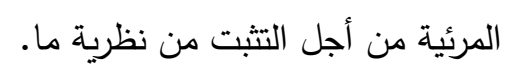

\section{خطوات استكثاف المعرفة من القواعد (KDD)Knowledge discovery in data):}

تتقيب البيانات هو العلم المختص بدراسة أساليب وطرائق إنتاج المعلومات وقات التواعد المعرفة من خلال

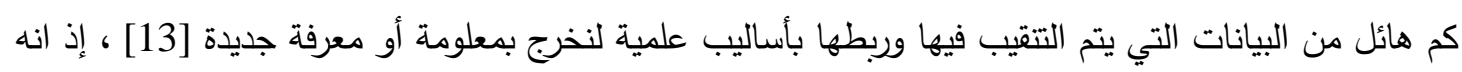

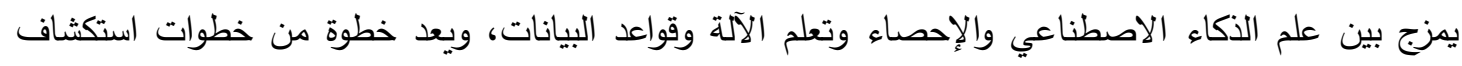

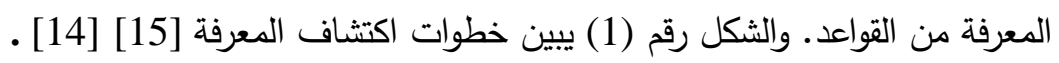

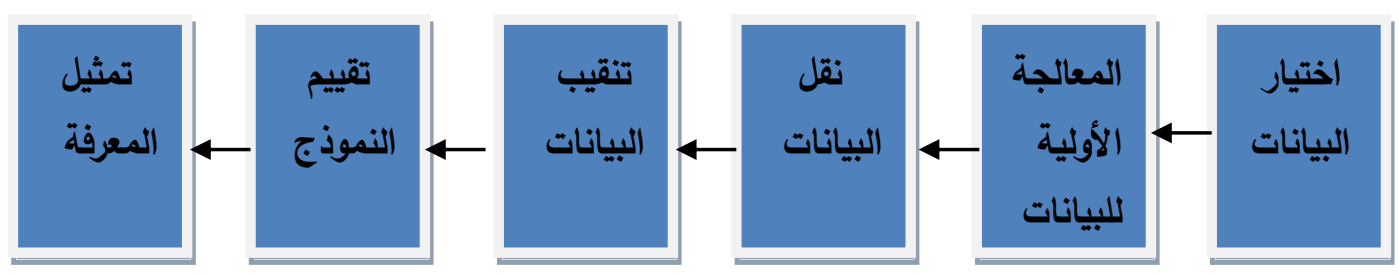

الثكل رقم (1) يبين خطوات اكتشاف المعرفة

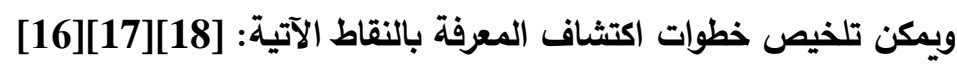

1. اختيار البيانات Data selection: في هذه المرحلة يتم تحديد واسترجاع البيانات الملائمة من مجموعة

البيانات.

2. المعالجة الأولية للبيانات من اجل تهيئتها Data preprocessing: وهي مرحلة تجهيز وعزل البيانات

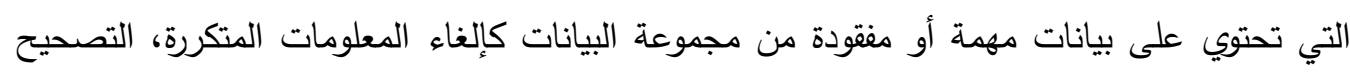

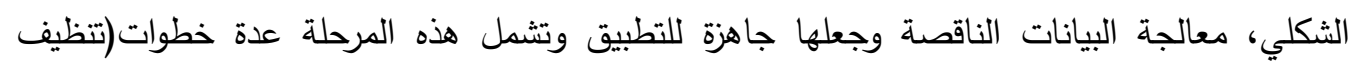

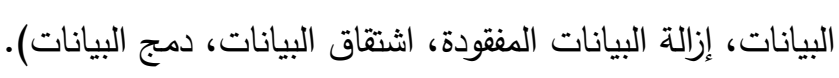

3. نقل البيانات Data transformation: وهي عملية نقل البيانات التي تم اختيارها إلى شكل ملائم

$$
\text { لإِجراءات البحث والاسترجاع. }
$$


4. تتقيب البيانات Data mining: في هذه المرحلة سيتم تطبيق أسلوب ذكي لاستخراج نماذج مفيدة قدر الإمكان مثل استخدام الثبكات العصبية بأنواعها المختلفة إذ يتم تدريب الشبكة على نمط معين واختبارها

$$
\text { على أنماط مشابهة. }
$$

5. تتييم النموذج Pattern evaluation: بعد استخراج النماذج المهمة والتي تمثل المعرفة يتم تقييمها

استتاداً إلى مقاييس محددة مثلاً استخدام الطرق الإحصائية وسيتم ذكرها لاحقاً.

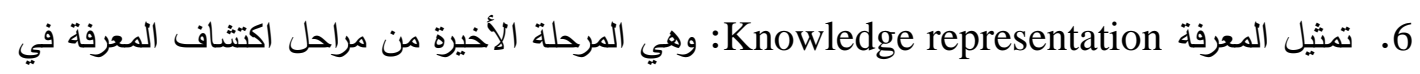
القواعد وهي المرحلة التي يراها المستفيد، وهذه المرحلة الأساسية تستعمل الأسلوب المرئي لمساعدة المستفيد في فهم وتقسير نتائج استخراج البيانات.

أهم التقنيات المستعملة في التنقيب

1. التنقيب باستعمال أدوات الاستعلام وتقنيات الإحصائيات: وهي عملية تحليل بدائي للبيانات، ويجب أن يتم أنمات

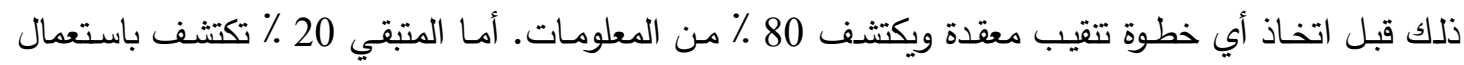
تقنيات التتقيب الأخرى. [19][20]

2. الإظهار visualization: وهي طريقة جيدة في استخلاص عينات من مجموعة المعطيات، تطبق في بداية عملية تحري المعطيات، لأنها تعطي فكرة جيدة حول كفاية المعطيـات والعينات التي يمكن استخلاصها منها.

3. الثبكات العصبية Neural Networks. خوارزمية الثبكة العصبية تثبه في تركيبتها تركيبة مخ الإنسان،

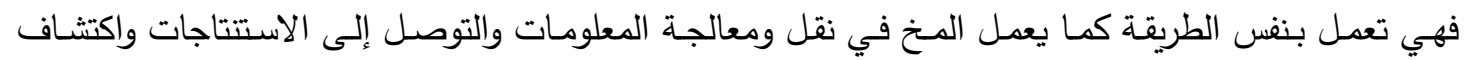

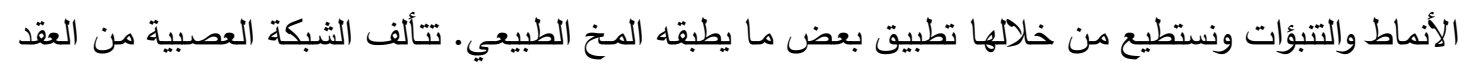
(التي تتاظر الخلايا العصبية) والروابط التي تصل بينها (التي تتاظر الوصلات العاتئية العصبية). قد تحتوي خوارزمية

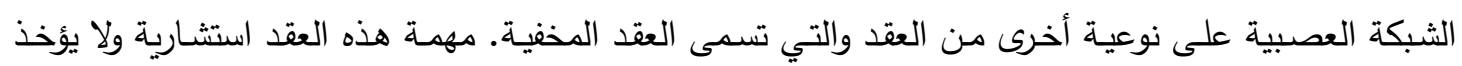
بقيمها إلا بعد أن يتم اعتماد استشارتها في حالة صحتها وبعد التجربة الفعلية. وكلما تم تطبيق الخوارزمية يتم تطوير وتحديث العقد الأصلية بأن تأخذ بالاعتبار قيم العقد المخفية المناسبة والتي تدعم الحصول على نتائج

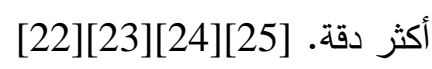

4. استقراء القاعدة Rule Induction: أن تقنية استقراء القاعدة من التقنيات الأساسية في التتقيب في البيانات وأكثرهـا شيوعا في مجال استكثاف المعرفة، وهي أقرب ما تكون إلى مـا يسمى بعملية التتقيب بحد ذاتها، والشيء الأساس في هذه الحالة هو القاعدة. تبين هذه القاعدة ما يجري داخل القاعدة وتظهر لنا ما لم نكن نعرفه

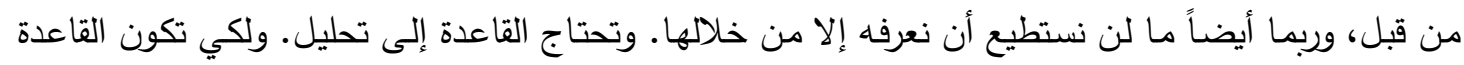
مكتملة وذات فائدة، فإنه يلزم لها تقييم، وهو عبارة عن نوعين إضافيين من المعلومات التي يجب أنسات أن تلازمها،

وهذه المعلومات الإضافية هي: [23]

الصحة Accuracy : كم هي نسبة صحة القاعدة (وقوع النتيجة في حال وقوع السبب). • التغطية Coverage : كم نسبة السجلات المحققة للقاعدة إلى كافة السجلات في قاعدة البيانات.

5. التحليل التسلسلي Sequential analysis: يعد التحليل التسلسلي من أهم تقنيات التتقيب في البيانات، نظراً للنتائج الدقيقة التي يتم التوصل إليها باستعمال هذه الطريقة لإمكانية تطبيقها في حل العديد من المشاكل

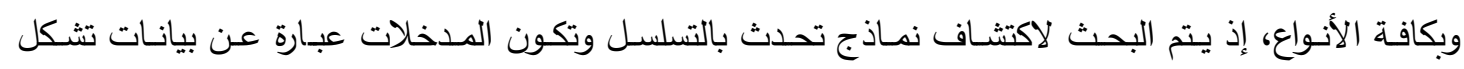


مجموعة متسلسلة وكل سلسلة من البيانات هي قائمة منظمة من العمليات. وتم اعتماد هذه الطريقة في البحث وسيتم توضيحها لاحقاً.

فإذا توصلت إحدى هذه التقنيات إلى إيجاد شكل مناسب، فبإمكان بقية التقنيات إيجاد هذا الثكل

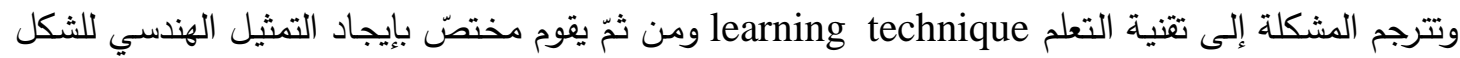
الناتج• [24]

نظريـة قواعد تركيب الفضاءات وتحديد الأهمية التركيبية نظرية قواعد تركيب الفضاءات هي طريقة حسابية في تحليل النظم الهندية وتركز على الجوانب العلائقية للفضاء يتم من خلالها حساب قيم عدم التتاظر النسبي المعدلة حسب المعادلات التالية: معدل عمق الفضاء = مجموع عدد الخطوات التي يبعدها الفضاء عن أي من فضاءات النظام الاخرى / (عدد

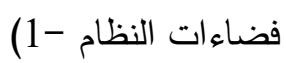
عدم التناظر النسبي للفضاء = 2*(معدل عمق الفضاء-1)/ (عدد فضاءات النظام-2) عدم التناظر النسبي المعدلة = عدم التتاظر النسبي للفضاء / عدم التتاظر النسبي للفضاء الأساسي من مخطط النطاء العمق وتكون قيم عدم التتاظر النسبي المعدلة الناتجة من هذه المعادلة حول العدد 1، وتثير القيم الأقل من 1 إلى

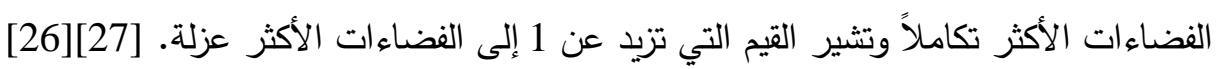
وفي هذا البحث كان عدد فضاءات النظام 30 فضاء، ومستوى العمق هو 6 (حسب خاصية الاتصالية بين الفضاءات) كما سيوضح لاحقاً.

ولتتفير العلاقة بين الفضاءات المختلفة الموجودة وتحديد الأهية التركيبية فإن : [29] [28]

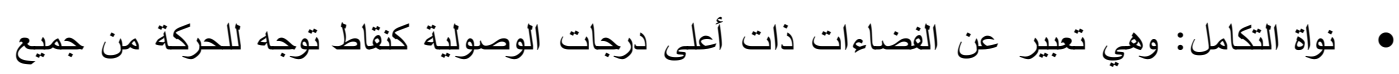

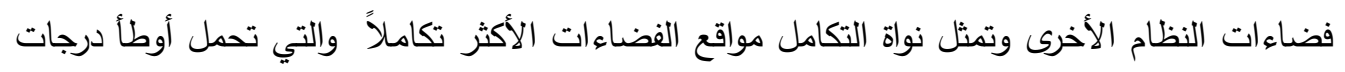
عدم التناظر النسبي الحقيقي. • نواة العزل: وهي تعبير عن أكثر الفضاءات عزلة واقلها وصولية كنقاط توجه للحركة من جميع

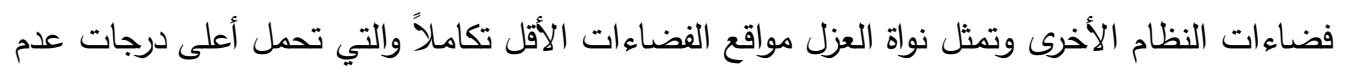
التناظر النسبي الحقيقي. المواد وطرائق العمل: - المز تم في هذا البحث إيجاد التحليل التسلسلي بحكم الموقع لكل نموذج في البيانات لغرض تعريف القيمة النوعية للنموذج والتي هي تمثيل للعلاقات الفضائية المكونة لمخطط احد المباني ومثل هذا الأمر يساهم في ندي تحديد درجة الأهمية الفضائية موضعياً وشمولياً للنموذج ضمن المنظومة، تم التعامل مـع مخطط أفقي هندسي لأحد المدارس المكونة من طابقين كما موضح ذلك في الثكل رقم(2) ، وبغية إيجاد تحليل منطقي لعملية

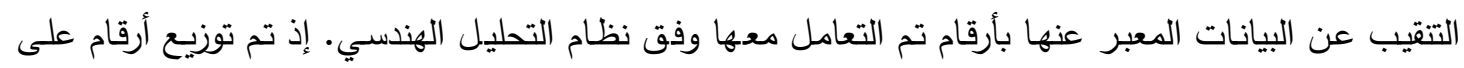
الفضاءات الموجودة كما موضح ذلك في الثكل رقم(3) بداية من الرقم 1 ووجد أن عدد الفضاءات الموجودة يساوي 30. 


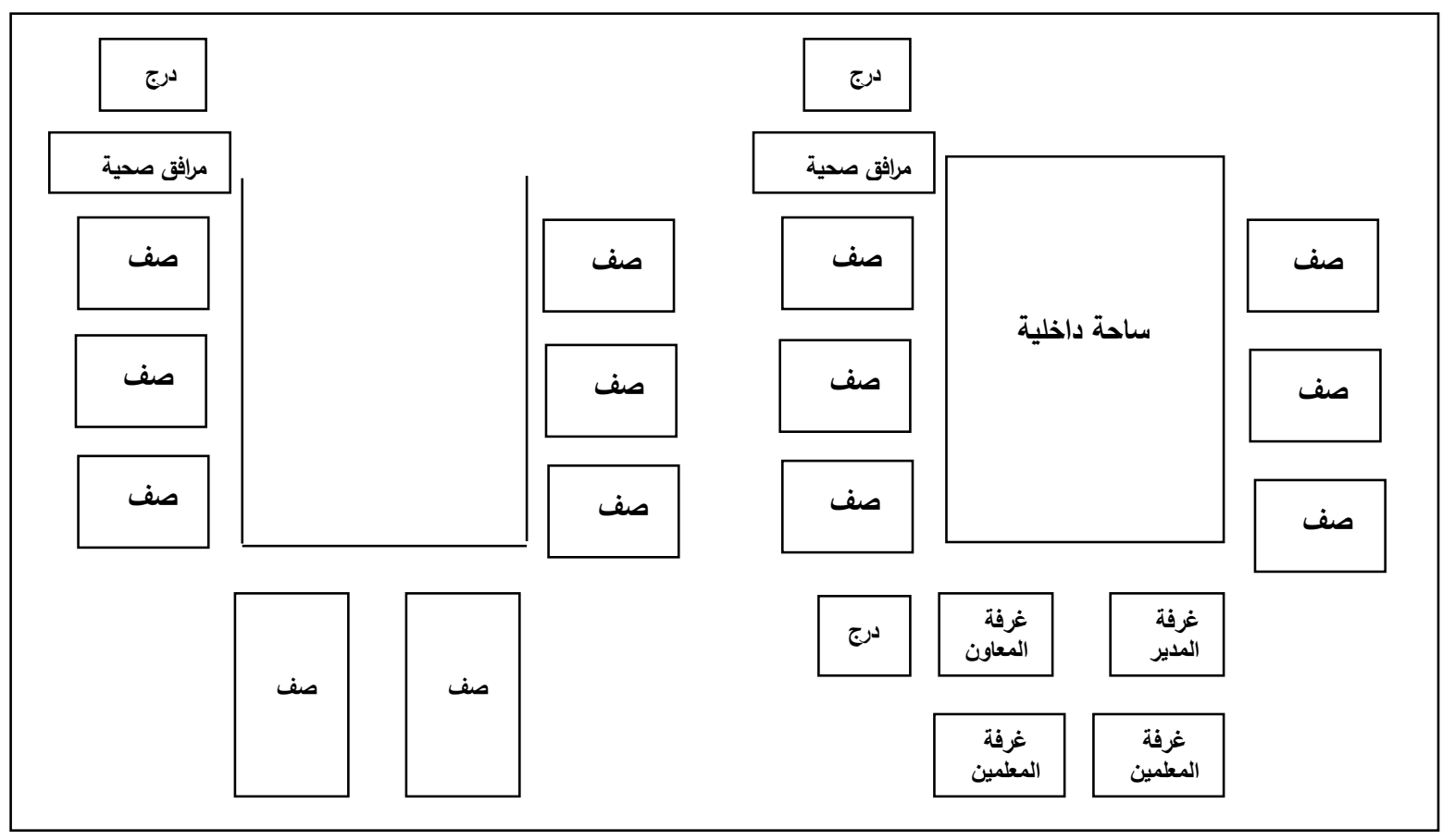

ب- مخطط الطابق الأول

أ- مخطط الطابق الأرضي

الثكل رقم (2) يبين مخطط أفقي هندي لأحد المدارس المكونة من طابقين

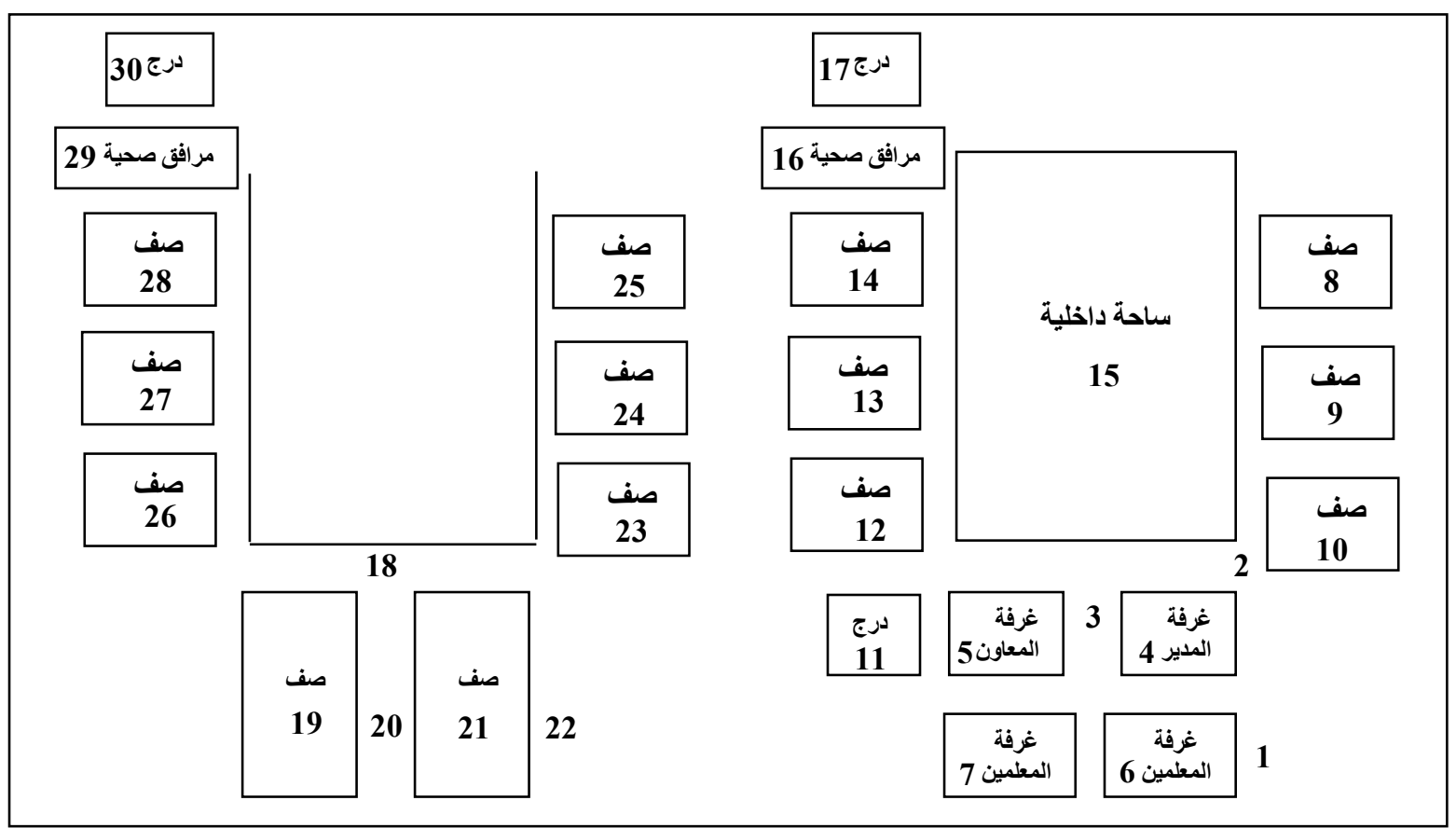

ب- ب مخطط الطابق الأول

أ- - مخطط الطابق الأرضي

الثكل رقم (3) يبين مخطط أفقي هندسي لأحد المدارس المكونـة من طابقين بعد توزيـع الأرقام على الفضـاءات (إذ أن عدد الأد

الفضاءات في الدرسة كان 30 فضاء) 
ويمكن القول أن هناك عدة تطبيقات هامة ترتبط بالتتظيم الفضائي الذي هو تعبير ناجح عن العلاقات

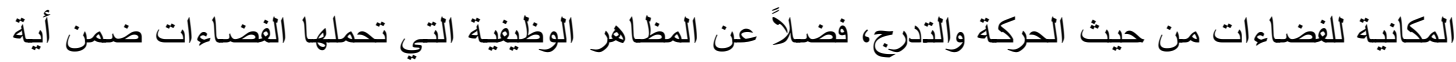

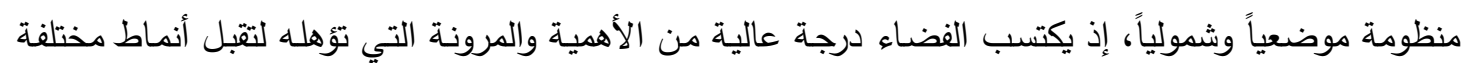
من صيخ الاستعمال التي يتواكب من خلالها مع المحيط. مصطلح الفضاء هو تعبير عن الحيز المكاني(الثكل الهندسي) للغرفة أو الممر ضمن المخطط الأفقي وهو مستعمل بشكل واسع في مجال الهندسة وتصميم الأبنية.

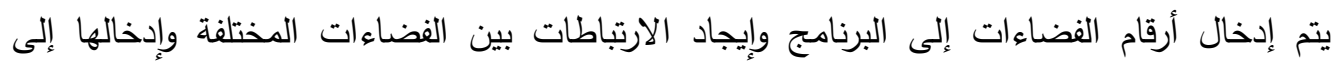
البرنامج ويتم حساب قيم عدم التتاظر النسبي المعدلة. ويتحقق عدم التناظر النسبي من خلال العمق في علاقات

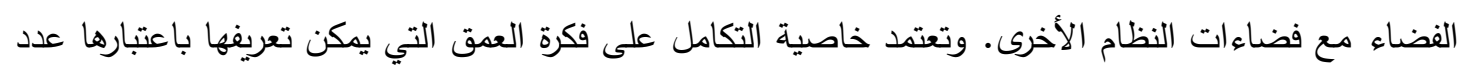
الخطوات من عقدة معينة إلى كل العقد الأخرى. تعتبرخاصية الاتصالية التي تم توضيحها في الثكل رقم (4) الخاصية التركيبية الأكثر وضوحًا بالنسبة

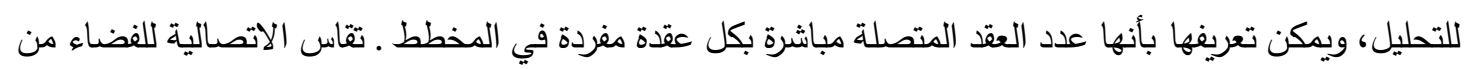
مجموع الفضاءات الأخرى التي ترتبط به أو تتقاطع معه أي التي تبعد عنه بخطوة واحدة. وتتراوح قيم عدم التتاظر النسبي الدعدلة التي تم حسابها في البرنامج حول الرقم الواحد، إذ تشير القيم التي تقل عن الواحد إلى تكامل الفضاء ضمن النظام (التي تحمل اقل قيم من عدم التتاظر النسبي المعدلة )، بينما تثير القيم المقاربة للواحد والتي تزيد عنه إلى فضاءات معزولة (التي تحمل أعلى قيم من عدم التناظر النسبي المعدلة).

يتم تحديد أهمية الفضاء بالاعتماد على قيمة درجة عدم التتاظر النسبي المعدلة إذ أن اقل قيمة من قيم درجة عدم التتاظر النسبي المعدلة يعتبر أفضل فضاء في حين أن أعلى قيمة من قيم درجة عدم التناظر النسبي المعدلة يعتبر أسوء فضاء. 


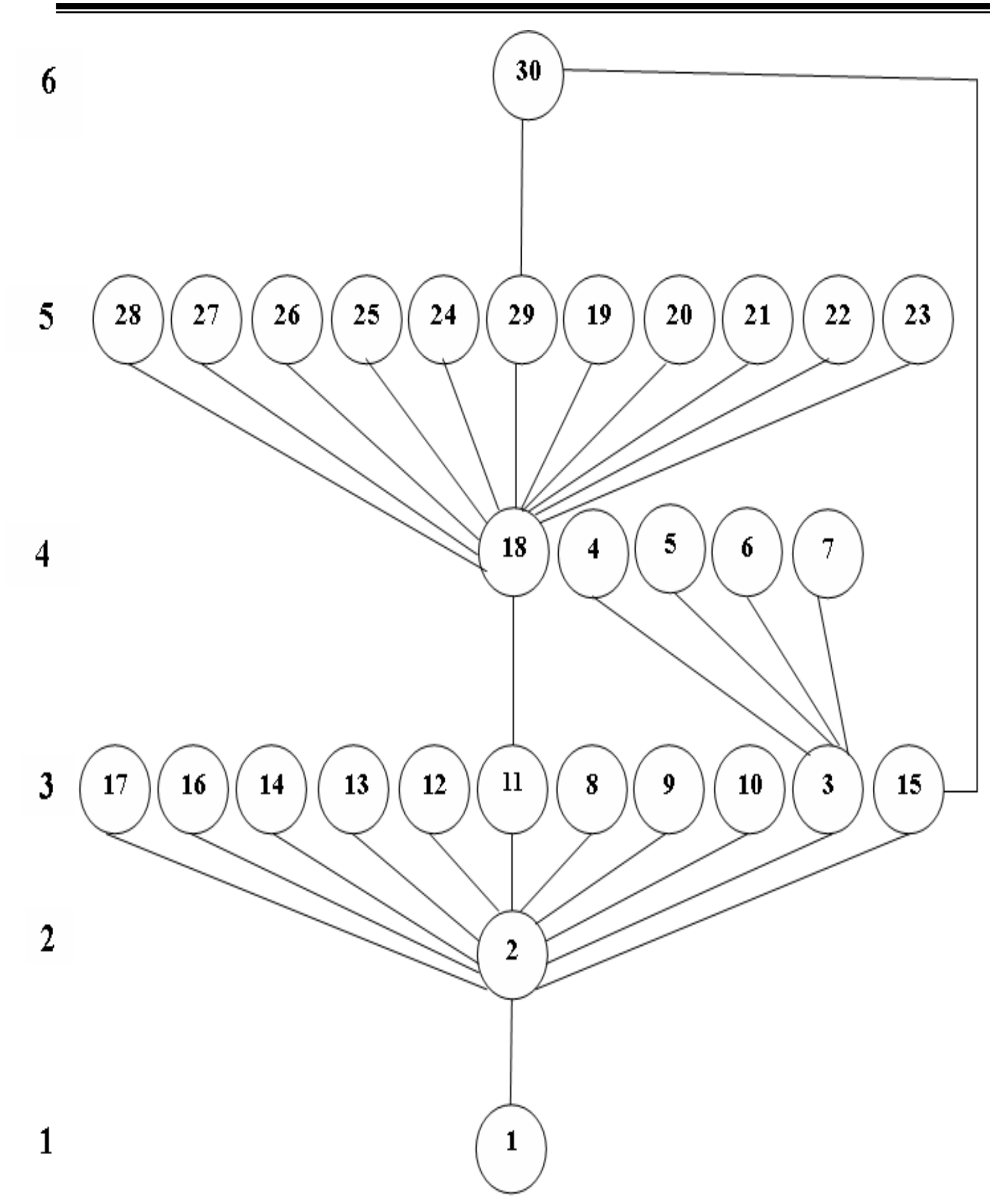

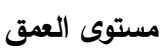

الثكل رقم (4) يبين خاصية الاتصالية الخاصة بالمخطط الهندسي 
لتحقيق عملية التتقيب عن البيانات تم في هذا البحث إتباع طريقة التحليل التسلسلي(كما تم ذكره سابقاً) بحكم الموقع لكل نموذج في البيانات لغرض تعريف القيمة النوعية للنموذج والتي هي تمثيل للعلاقات الفضائية،

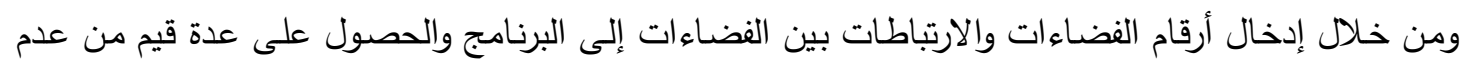

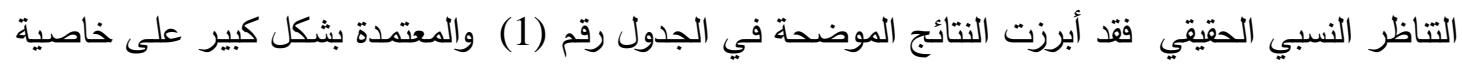

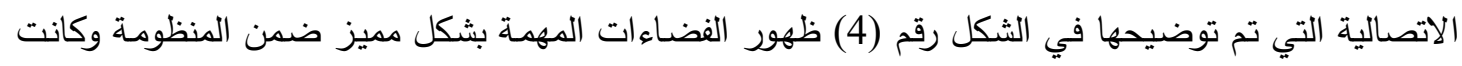
النتائج موافقة للواقع المنطقي من خلال تحليل أرقام الفضاءات الموجودة في الثكل رقم (3)، إذ تم تح تحديد أهمية

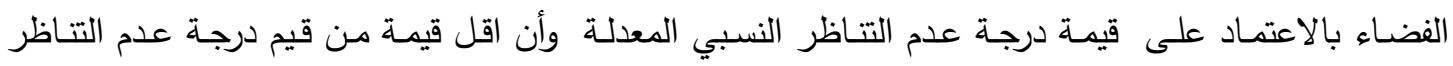

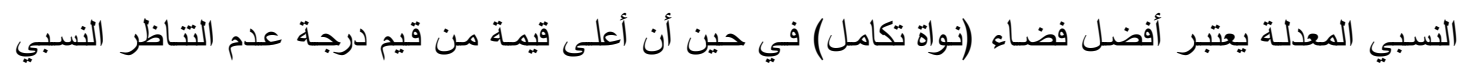
المعدلة يعتبر أسوء فضاء (نواة عزل). تم الوصول إلى أن الفضاء رقم 2 (الذي يمثل البهو (موزع الحركة للطابق الأرضي)) هو أفضل فضاء

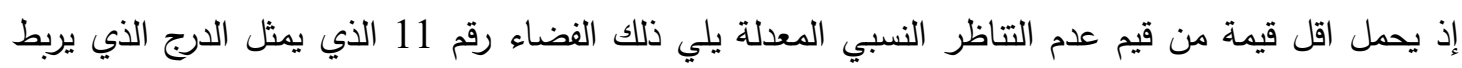
الطابق الأرضي بالطابق الأول ثم الفضاء رقم 18 (الذي يمثل البهو (موزع الحركة للطابق الأول)) وهكذا بالنسبة

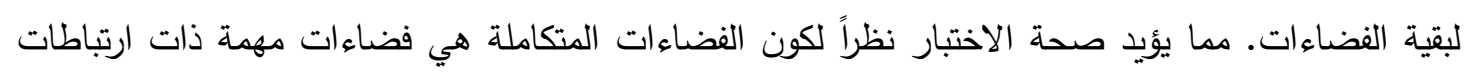
واتصالات مميزة على مستوى المنظومة. ونظراً لقلة الدراسات العربية المعنية بعملية التتقيب عن البيانات فان هذا البحث يعد خطوة نحو الأمام في مجال توضيح علاقة عملية التنقيب عن البيانات بنظرية قواعد تركيب الفضاء الخاصة بتحليل جوانب لنبل هندسية وتخطيطية على مستوى المباني والمنظومات الحضرية وصولاً إلى المقياس الأكبر للمدينة وما يرتبط به توله

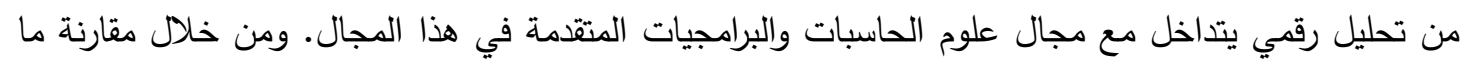
توصل إليه البحث مع الدراسات السابقة التي تتاولت موضوعات ذات صلة بهذ بذات التوجه فان الإضافة المعرفية تكمن في ربط الجوانب الإدراكية والحسية للمتغيرات وتحويلها إلى صيخ رقمية يمكن معالجتها حاسوبياً. 
الجدول رقم (1) يبين النتائج التي تم الحصول عليها بعد تطبيق البرنامج وتحديد أهمية الفضاء

\begin{tabular}{|c|c|c|c|}
\hline رقم الفضاء & الاتصالات حسب عدد & قيم عدم التناظر النسبي المعدلة & تعلى قيم أهمية الفضاء بالاعتماد التناظر النسبي \\
\hline 1 & 1 & 0.702 & 5 \\
\hline 2 & 12 & 0.381 & 1 \\
\hline 3 & 5 & 0.653 & 4 \\
\hline 4 & 1 & 1.034 & 10 \\
\hline 5 & 1 & 1.034 & 10 \\
\hline 6 & 1 & 1.034 & 10 \\
\hline 7 & 1 & 1.034 & 10 \\
\hline 8 & 1 & 0.762 & 7 \\
\hline 9 & 1 & 0.762 & 7 \\
\hline 10 & 1 & 0.762 & 7 \\
\hline 11 & 2 & 0.435 & 2 \\
\hline 12 & 1 & 0.762 & 7 \\
\hline 13 & 1 & 0.762 & 7 \\
\hline 14 & 1 & 0.762 & 7 \\
\hline 15 & 2 & 0.708 & 6 \\
\hline 16 & 1 & 0.762 & 7 \\
\hline 17 & 1 & 0.762 & 7 \\
\hline 18 & 12 & 0.490 & 3 \\
\hline 19 & 1 & 0.871 & 9 \\
\hline 20 & 1 & 0.871 & 9 \\
\hline 21 & 1 & 0.871 & 9 \\
\hline 22 & 1 & 0.871 & 9 \\
\hline 23 & 1 & 0.871 & 9 \\
\hline 24 & 1 & 0.871 & 9 \\
\hline 25 & 1 & 0.871 & 9 \\
\hline 26 & 1 & 0.871 & 9 \\
\hline 27 & 1 & 0.871 & 9 \\
\hline 28 & 1 & 0.871 & 9 \\
\hline 29 & 2 & 0.816 & 8 \\
\hline 30 & 2 & 0.702 & 5 \\
\hline
\end{tabular}

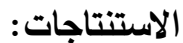

تم التوصل من خلال البحث إلى جملة من الاستتتاجات يمكن إجمالها بما يلي: تقنيات التتقيب عن البيانات تجيب على العديد من الأسئلة، وفي وقت قياسي، بخاصة تلكئك النوعية من بن الأسئلة التي كان من الصعب الإجابة عليها، إن لم يكن مستحيلاً، باستعمال تقنيات الإحصاء

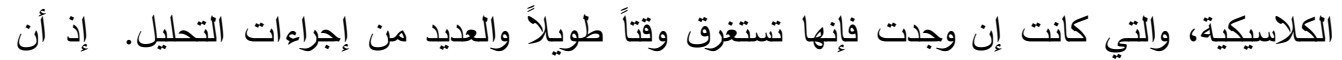
عملية التتقيب عن البيانات تستغرق وقت اقل نسبياً في تحليل المخطط الهندسي الخاص بالمدرسة وإيجاد أفضل الفضاءات مقارنة مع الطرق التقليدية الأخرى. 
تركز تقنيات التنقيب المختلفة على بناء واستكثاف السلوك والاتجاهات، مما يسهح بتقدير القرارات الصحيحة واتخاذها في الوقت المناسب.

التتقيب عن البيانات هي عملية استخراج المعرفة من كمية كبيرة من البيانات (مستودعات البيانات)، إذ إذ الذان

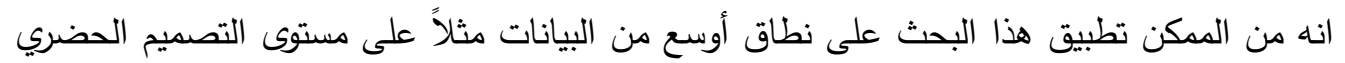
وتخطيط المدن التي تضم بيانات كبيرة نسبياً للخروج بمؤشرات منطقية وذات مصني منداقية التهات في التحليل تدعم فكرة البحث. وذلك باستعمال أساليب جديدة سواء أكانت الخوارزميات أم أساليب الذكاء الاصطناعي وبمراحل متعددة، ومن خلال ذلك فقد استتتج البحث انه يمكن الوصول إلى النتائج بوسائل مختلفة ضمن نفس الإطار العام ومنها استعمال أسلوب التحليل التسلسلي للبيانات.

قواعد تركيب الفضاء هي وسيلة قياس الغاية منها اكتثاف الخصائص الاجتماعية لأي مجتمع من خلال

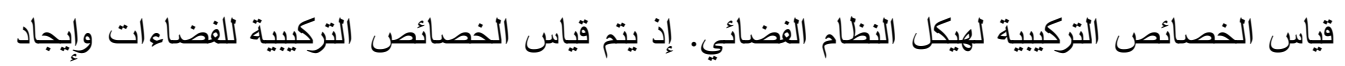
نواة التكامل والتي تمثل مواقع الفضاءات الأكثر تكاملاً والتي تحمل أوطأ درجات عدم التكامل النسبي لتئي

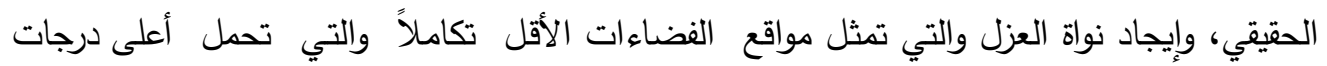
عدم التكامل النسبي الحقيقي لمخطط أفقي هندسي لأحد المدارس المكونة من طابقين، وعلى هذاء الأساس تم تحليل المخطط وإبراز مواطن القوة والضعف على مستوى التحليل الهندسي للفضاءات. أظهرت نتائج التحليل أن الفضاءات الهندسية ضمن المخطط الهندسي للمدرسة التي تحمل أهمية كبيرة

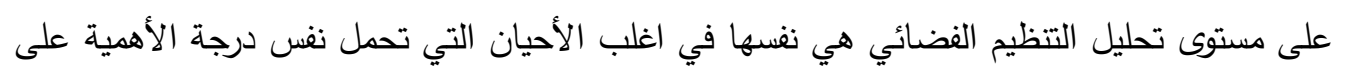

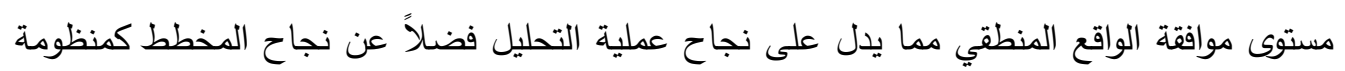
هندسية وظيفية. من خلال البحث تم الوصول إلى أن الفضاء رقم 2 (الذي يمثل البهو (موزع الحركة

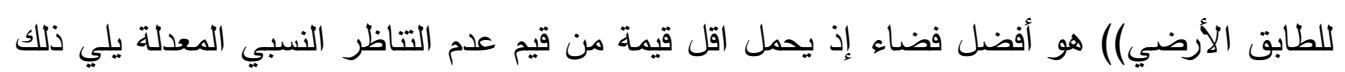
الفضاء رقم 11 الذي يمثل الدرج الذي يربط الطابق الأرضي بالطابق الأول ثم الفضاء رقم 18 (الذي

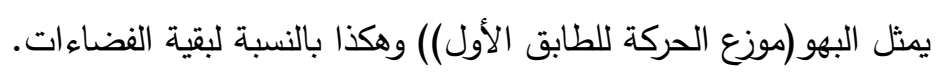

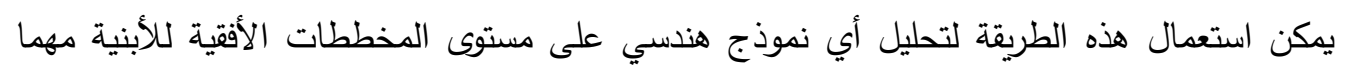
اختلفت وظائفها وتتييم مستويات نجاحها فيما يتعلق بالعلاقات الفضائية التي تعمل كمنظومة متكاملة يتم من خلالها تحديد نجاح أو فشل المخطط الهندسي.

\section{المصادر}

[1] Firdhous, M. F., (IJACSA) International Journal of Advanced Computer Science and Applications, 1(6): 9-16 (2010)

[2] Felista, Eze and Adeoye Olufemi, International Journal of advanced studies in Computer Science and Engineering IJASCSE, 3(2): 28-37(2014)

[3] Rehman, Nelofar, International Journal of Computer Science and Mobile Computing IJCSMC, 6(7): 227-231 (2017)

[4] Ismaeel, Ayad G. and Dina Y. M. , (IJCSA) International Journal of Advanced Computer Science and Applications, 7(7): 69-76 (2016)

[5] Qiu, Manying, and Steve Davis, Issues in Information System, 4(1): 254-260 (2004)

[6] Sahu, Pankaj and Arif Khan, International Journal of Engineering Sciences \& Management, 2(1): 65-72 (2012) 
[7] Pei, Jian, IEEE Transactions on knowledge and data Engineering, 16(10): 1-16 (2004)

[8] Abdellatif,T. and Mohammed A., International Journal of Computer, 8(2): 161174 (2011)

[9] Venkatadri, M., International Journal of Computer, 15(7): 19-22 (2011)

[10] Mohd. Shahnawaz, Ashish Ranjan, International Journal of Engineering and Advanced Technology (IJEAT), 1(1): 20-24 (2011)

[11] Sharma, Sugam, and Shashi Gadia, International Journal of Computer Science \& Engineering Survey (IJCSES) 1(1): 3-37 (2010)

[12] Manchandia, Karan and Navdeep Khare, International Journal of Engineering Sciences \& Research Technology, 6(3): 424-430 (2017)

[13] Ullah, Irshad, International Journal of Computer, 2(6): 127-136 (2010)

[14] Baradwaj, Brijesh Kumar, (IJACSA) International Journal of Advanced Computer Science and Applications, 2(6): 63-69 (2011)

[15] Mohamed, Nashaat and Ahmed Shawky El-Bhrawy, Journal of Computer Engineering, 18(6): 55-59 (2016)

[16] Sathyamoorthy, S., International Research Journal of Engineering and Technology (IRJET), 2(9): 431-434 (2015)

[17] Ramamohan, Y. and K. Vasantharao, International Journal of Soft Computing and Engineering (IJSCE), 2(3):11-194 (2012)

[18] Sahu, Hemlata and Shalini Shrma, International Journal of Computer Technology and Electronics Engineering (IJCTEE) 1(3): 114-121 (2012)

[19] Allison, L., Conferences in research and practice in information, 16: 1-8 (2003)

[20] Arif, Muhammad and Mehdi Hussain, International Journal of Database Theory and Application, 8(1): 245-270 (2015)

[21] Srinivas, K. and B. Kavihta R., International Journal on Computer Science and Engineering, 2(2): 250-255 (2010)

[22] Hebah H. , International Journal of Web Applications, 1(4): 183-190 (2009)

[23] Padhy, Neelamadhab and Dr. Pragnyaban Mishra, International Journal of Computer Science, Engineering and Information Technology (IJCSEIT), 2(3): 43-58 (2012)

[24] Singhal, S. and Monika J., International Journal of Innovative Technology and Exploring Engineering (IJITEE), 2(6): 250-253 (2013)

[25] Guan, Yanpeng, Chemical Engineering Transactions AIDIC, 805-810 (2017)

[26] Shen, YING, SPIE Digital Library, 7144: 1-12 (2008)

[27] Al-Juboori, Udai and Faris Ali, International Transaction Journal of Engineering, 5(3): 143-165 (2014)

[28] Zimniak, Marcin and Janusz R. Getta, International Journal of Database Theory and Application, 6(6): 63-74 (2013)

[29] Narvaeza, Laura and Alan P., International Transaction Journal of Engineering, Management, \& Applied Sciences \& Technologies, 3(3): 293-309 (2012) 